\title{
Die Besteuerung von Alimentenleistungen - ein Thema mit Praxisrelevanz
}

\section{Daniel Zenhäusern}

Diplomierter Wirtschaftsprüfer

Korrespondenz:

Daniel Zenhäusern

FMH Treuhand Services

Zenhäusern Treuhand AG

Kantonsstrasse 39

CH-3930 Visp

Tel. 0279489019

Fax 0279489021

daniel.zenhaeusern@

fmhtreuhand.ch

\section{Einleitung}

Eine Scheidung kostet Geld, sehr viel Geld! 2007 lag die Scheidungsquote in der Schweiz bei 49,1\%; und laut Studien soll die Scheidungsrate bei Ärzten überdurchschnittlich hoch sein.

Neben vielen anderen Problemen gilt es auch, eine für alle Beteiligten (Ehepartner und Kinder) gute finanzielle Regelung der Alimentenleistungen zu finden.

Was ist aber, wenn die Kinder volljährig sind, und die Alimente nicht mehr vom steuerbaren Einkommen abgezogen werden können, wie dies ein kürzlich veröffentlichter Bundesgerichtsentscheid [1] bestätigt hat?

\section{Was sind Alimente?}

Alimente sind Unterhaltsbeiträge, die vom Richter aufgrund familienrechtlicher Verpflichtungen bei Scheidung, gerichtlicher oder tatsächlicher Trennung festgelegt werden. Dabei unterscheidet man grundsätzlich zwischen «Scheidungsalimenten» und «Kinderalimenten».

\section{Steuerliche Behandlung der Alimente}

Aus steuerlicher Sicht gilt es zu unterscheiden, ob die Alimentenleistungen als periodische Rente oder als einmalige Kapitalleistungen bezahlt werden.

Scheidungsalimente werden an den geschiedenen Ehegatten bezahlt. Beim Empfänger werden die Alimente zum übrigen Einkommen hinzugerechnet und versteuert. Der Schuldner kann die Scheidungsalimente vollumfänglich vom steuerbaren Einkommen in Abzug bringen. Diese Regelung gilt sowohl beim Bund als auch bei allen Kantonen [2].

Kinderalimente an Minderjährige werden demjenigen Elternteil zum übrigen Einkommen hinzugerechnet und versteuert, der über das Sorgerecht für die Kinder verfügt. Der Kinderabzug kann demnach auch von diesem Elternteil vorgenommen werden. Vorbehalten bleiben Spezialregelungen bei alternierender Obhut der Kinder. Der Schuldner kann die Unterhaltsbeiträge vom steuerbaren Einkommen in Abzug bringen. Ein Kinderabzug ist beim Schuldner somit nicht möglich.
Kinderalimente an volljährige Kinder sind beim Empfänger nicht steuerpflichtig und können darum beim Schuldner auch nicht zum Abzug zugelassen werden. Der Kinderabzug ist beim Schuldner der Alimentenleistungen zulässig, wenn das Kind in der beruflichen Erstausbildung steht und unterstützungspflichtig ist. Als unterstützungspflichtig gilt es, wenn das eigene Einkommen (Lohn, Ersatzeinkommen, Stipendien usw., jedoch ohne Kinderalimente) eine gewisse Höhe nicht übersteigt. Die Höhe dieses Einkommens kann bei den einzelnen Kantonen unterschiedlich sein. Im Kanton Bern liegt diese Grenze beispielweise bei 18000 Franken pro Jahr [2].

Alimente in Form von Kapitalleistungen sind beim Empfänger steuerfrei und werden darum beim Schuldner auch nicht zum Abzug zugelassen. Der Kanton Wallis macht hier eine Ausnahme und behandelt diese Kapitalleistungen gleich wie Alimentenleistungen in Rentenform. Demnach sind diese im Wallis beim Begünstigten steuerbar (satzbestimmende Jahressteuer) und können daher auch beim Schuldner steuerlich in Abzug gebracht werden [3].

\section{Ein Beispiel aus der Praxis}

\section{Die böse Überraschung}

Ein Arzt, seit einigen Jahren geschieden, macht wie gewohnt in seiner Steuererklärung die bezahlten Unterhaltsbeiträge an seine Ex-Frau und die vier Kinder (14, 15, 17 und 18 Jahre) von je 24000 Franken geltend. «Dank» dieser abzugsberechtigten Unterhaltszahlungen konnte das steuerbare Einkommen in den letzten Jahren von 320000 auf 200000 Franken reduziert werden. Die Liquidität des Arztes reichte aus, um noch etwas Steuerplanung zu betreiben. So zahlte er jährlich einen Beitrag von 6300 Franken in die Säule 3a ein, ausserdem konnte er sich noch einen jährlichen Einkauf von 30000 Franken in seine Pensionskasse leisten. Die Steuerbelastung belief sich damit auf etwa 48000 Franken pro Jahr. Nicht schlecht staunte der Arzt, als er die Steuerveranlagung erhielt. Die Steuerverwaltung hatte eine Aufrechnung von Unterhaltsbeiträgen für das volljährige Kind von netto 17900 Franken 
vorgenommen. Die Veranlagungsbehörde akzeptierte statt der Alimentenleistung von 24000 Franken lediglich den Kinderabzug von 6100 Franken für das volljährige Kind. Die Steuerbelastung belief sich somit statt der geplanten 48000 neu auf 55000 Franken pro Jahr.

Alle Kinder des Arztes haben die Absicht, ein Hochschulstudium zu absolvieren. Der Arzt wird für die Unterhaltszahlungen an die Kinder also noch einige Jahre aufkommen müssen.

\section{Die Ernüchterung}

Für den Arzt wird die Steuerbelastung künftig massiv zunehmen, da er die Unterhaltsbeiträge an die volljährigen Kinder nicht mehr von den Steuern in Abzug bringen kann.

\section{Der kleine Trost}

Immerhin kann der Arzt den Kinderabzug von je 6100 Franken pro Kind in Abzug bringen.

\section{Fazit}

Alimentenleistungen an Kinder in Ausbildung belasten die Liquidität über Jahre hinweg enorm. Dabei muss berücksichtigt werden, dass nach Erreichung der Volljährigkeit die effektiven Unterhaltsbeiträge nicht mehr vollumfänglich vom steuerbaren Einkommen in Abzug gebracht wer- den können. Somit fallen, nebst den monatlichen Alimentenleistungen an die volljährigen Kinder, auch höhere Steuern an. Bei gleichbleibenden Einnahmen und dem gewohnten Lebensstandard bleibt so für den Arzt wesentlich weniger Geld übrig. Der Handlungsspielraum für eine aktive Steuerplanung bleibt daher massiv eingeschränkt. Einkäufe in die Pensionskasse mit dem Ziel, die Steuerbelastung zu reduzieren und ein aktives Alterssparen anzustreben, bleiben oft während Jahren ein Wunschtraum. Die Mittel dafür sind schlichtweg nicht vorhanden.

Aus diesen Gründen ist eine sorgfältige persönliche Finanzplanung unerlässlich. Nur wer seine Ausgaben wirklich kennt und diese auch für die Zukunft richtig einplant, ist in der Lage, gezielte Einsparungen vorzunehmen und so eine aktive Steuer- und Pensionsplanung zu betreiben.

\section{Literatur}

1 BGE 2C_439/2007.

2 Art. 23 Bst f DBG; Art. 7 Abs. 1 StHG; Art. 33 Abs. 1 Bst. C DBG; Art. 9 Abs. 2 Bst. C StHG.

3 Art. 24 Bst. E DBG; Art. 7 Abs. 4 Bst. G StHG; Art. 33 Abs. 1 Bst. C DBG; Art. 9 Abs. 2 Bst. C StHG.; Art. 19 Bst. e StG VS, Art. 33 Abs. 1 StG VS. 